\title{
Assessment of Autonomic Function in Subjects Practicing Yoga Using Spectral Analysis and Approximate Entropy Method
}

Kamal Ahmed ${ }^{*}$

\author{
MET Department, Tennessee Tech University, Lewis Hall, Cookeville, TN, USA
}

${ }^{*}$ Corresponding author: Kamal Ahmed, Ph.D, SMIEE, MET Department, Tennessee Tech University

Box 5003, Lewis Hall, Cookeville, TN, USA 38501, E-mail: akamal@tntech.edu

Citation: Kamal Ahmed (2017) Assessment of Autonomic Function in Subjects Practicing Yoga Using Spectral

Analysis and Approximate Entropy Method. J Case Rep Stud 5(3): 304. doi: 10.15744/2348-9820.5.304

Received Date: March 07, 2017 Accepted Date: June 28, 2017 Published Date: June 30, 2017

\begin{abstract}
Objective: Autonomic nervous system involvement in subjects practicing Yoga has rarely been studied and has shown conflicting results. Our main purpose was to assess the effect of Yoga on autonomic function of group of subjects practicing Yoga regularly in comparison with another group with normal control subjects by measuring the frequency gain response of the two groups. Other aim of this study was to determine wither the duration of Yoga practicing correlated with measures of heart rate variability signal (HRV) using approximate entropy index (ApEn).

Methods: Fifty two subjects practicing Yoga and fifty one matched healthy subjects were evaluated at Yoga Center, Nashville, Tennessee, USA during 2014/ 2015 by measuring the breathing at different rates and HRV signal derived from Electrocardiogram (ECG). The auto-power and cross power spectra between HRV signal and breathing signal at different breathing rate were calculated and frequency gain response for both groups was obtained. ApEn described as measure of regularity of HRV was calculated for subjects practicing Yoga and healthy control subjects.

Results: Both frequency gain response and ApEn measure were reduced in normal subjects group in comparison with Yoga subjects group. The power spectra of Yoga practicing subjects showed increase in amplitude of high frequency (HF) value as well as higher amplitude of low frequency with respect to control subjects. On other hand, ApEn measure was significantly increased in Yoga subjects as they are practicing Yoga for long duration.

Conclusion: These findings suggest that the spectral analysis of HRV signal using breathing at different frequencies may detect balance of autonomic of autonomic system of Yoga subjects with specially with increasing the sympathetic activity (higher low frequency) and increasing also the parasympathetic tone (Higher frequency gain response) which can lead to balanced activity of Yoga subjects comparing to normal healthy subjects. APEn may be marker related to the yoga practicing duration for Yoga subjects.
\end{abstract}

Keywords: Heart Rate Variability; Gain Frequency Response; Approximate Entropy

\section{Introduction}

Yoga as practicing technique used since many centuries in East may improve the function in the autonomic nervous system in human being. However, this relationship has not been fully studied and some studies has conflicting results [1,2]. Several studies found more decrease cardiovascular mortality in subjects practicing Yoga than in control Healthy subjects who did not [3-6]. Recent attention has focused on the importance of long term pattern in heart rate i.e. Heart rate variability (HRV) to assess and quantify the autonomic function using simple statistical measures and power spectral analysis.

The aim of this study to investigate the auto power spectra of HRV signals at different breathing rates as well as producing the gain frequency response for both healthy subjects group (Control) and subjects practicing Yoga group. Another aim of this study is to introduce other statistics to assess the autonomic function developed from nonlinear dynamics. One such statistics, approximate entropy (ApEn) which quantifies the regularity of patterns in data set [7-16]. ApEn has been applied to HRV signal for both control and Yoga subjects.

\section{Material and Methods}

The study group was composed of fifty one healthy subjects $(25 \pm 8.7)$ years and fifty two Yoga Subjects (26 \pm 9.6$)$ years has been investigated in this study at Johns Hopkins hospital, MD, USA during 2013/2014. None of the Yoga subjects and normal healthy subjects had clinical signs of myocardial infraction, arterial hypertension, diabetes mellitus including type I and II or pulmonary disease. No drugs that could affect HRV parameters were used by the subjects and the control groups under study. The final group consisted of 52 Yoga practicing subjects and 51 of normal healthy subjects. All subjects agreed to participate in the research prior to their inclusion in the study and the consent of ethical committee was obtained and approved the study protocol. Each subject 
lies supine on a bed. The breathing signal is measured using a thermistor placed in the nose. The Electrocardiogram (ECG) is taken from wrists and ankle (Lead II). All the measurements were recorded for the duration of the experiments using laptop computer. After the subject has settled comfortably, a base line of the physiological measurements, ECG, derived HRV and breathing signal were measured for $5 \mathrm{~min}$ for both healthy subjects and patients with Yoga subjects at rest in supine position to calculate the ApEn and heart rate for each group. The subject is then asked to breathe deeply at different rates for 2 min following the light sequence, green inhale and red exhale. The cycle length is varied using frequency generator and the light indicator automatically divides the cycle length into $40 \%$ inhalation and $60 \%$ exhalation [10]. A rest period is allowed between each breathing rate sequence. The following breathing rates were examined for each subject 3,4,6,10,12,14,16,18,24 and 30 breath/min. Figure 1 shows the block diagram of the experiments.

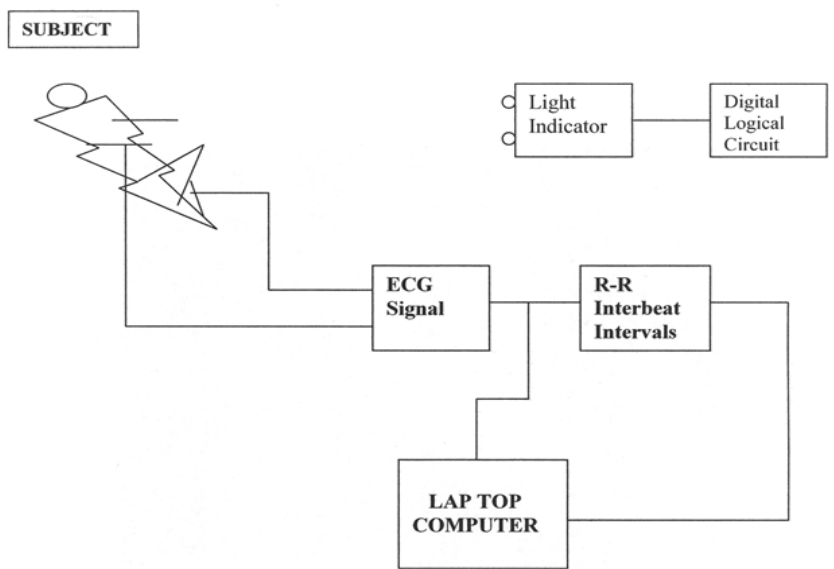

Figure 1: Block Diagram of Experimental Procedure

\section{Measurement and Analysis of Physiological Signals}

The ECG for every patient participated in this study is fed into an electronic device which detects the R-wave and measures the time until the next R wave occurs. Alternately, the peak of QRS was identified for each beat using rate-detector algorithm after exclusion of artifacts and ectopics. Those periods in which beat identification was poor were excluded from the analysis. This $\mathrm{R}-\mathrm{R}$ time is converted into voltage directly proportional to that time. The reconstructed voltage signals as shown in Figure 2 may now place as HRV signals and can be interfaced to Laptop computer to obtain auto power and cross power spectra of HRV signal and breathing signals at different rates using Fast Fourier transform (FFT) to calculate the gain frequency response and ApEn (Appendix).

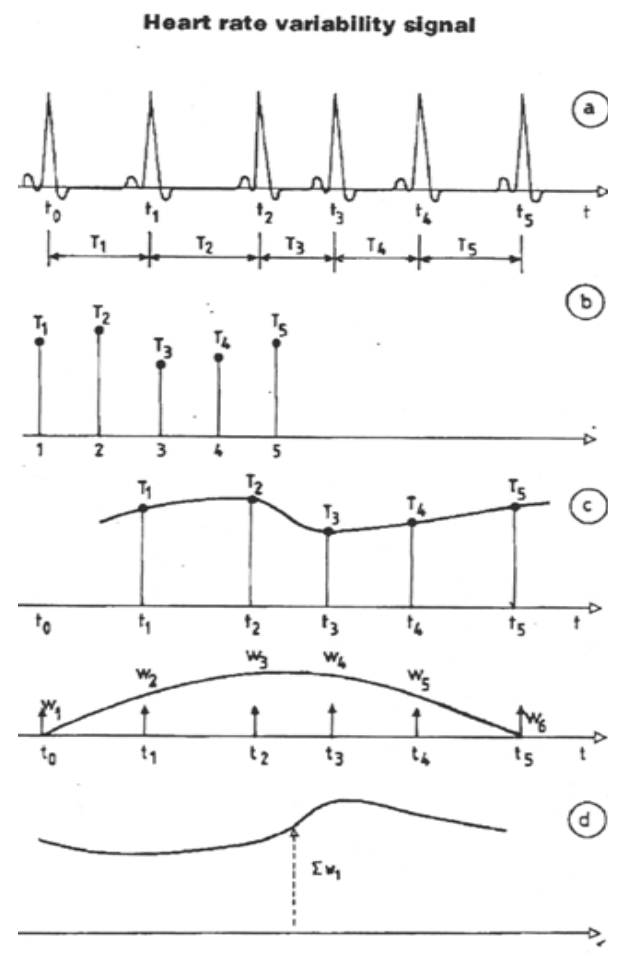

Figure 2: Derivation of heart rate variability (HRV) signal from Electrocardiogram (ECG) (a) ECG; (b) Detection of R-R interval; (c) Construction of HRV signal and (d) Smoothed Derivation HRV signal 


\section{Identification Procedure of Frequency Gain Response}

Consider a linear system with an input-output relationship as shown in Figure 3. It can be represented by a transfer function which can be found by several conventional methods e.g. impulse response, $H(j w) . R(j w)$, where $H(j w)$ and $R(j w)$ are the Fourier transform (using FFT algorithm) of the output signal $\mathrm{h}(\mathrm{t})$ and input signal $\mathrm{r}(\mathrm{t})$, respectively. If we define the transfer function between $\mathrm{H}(\mathrm{jw})$ and $\mathrm{R}(\mathrm{jw})$ as shown in figure 3 as:

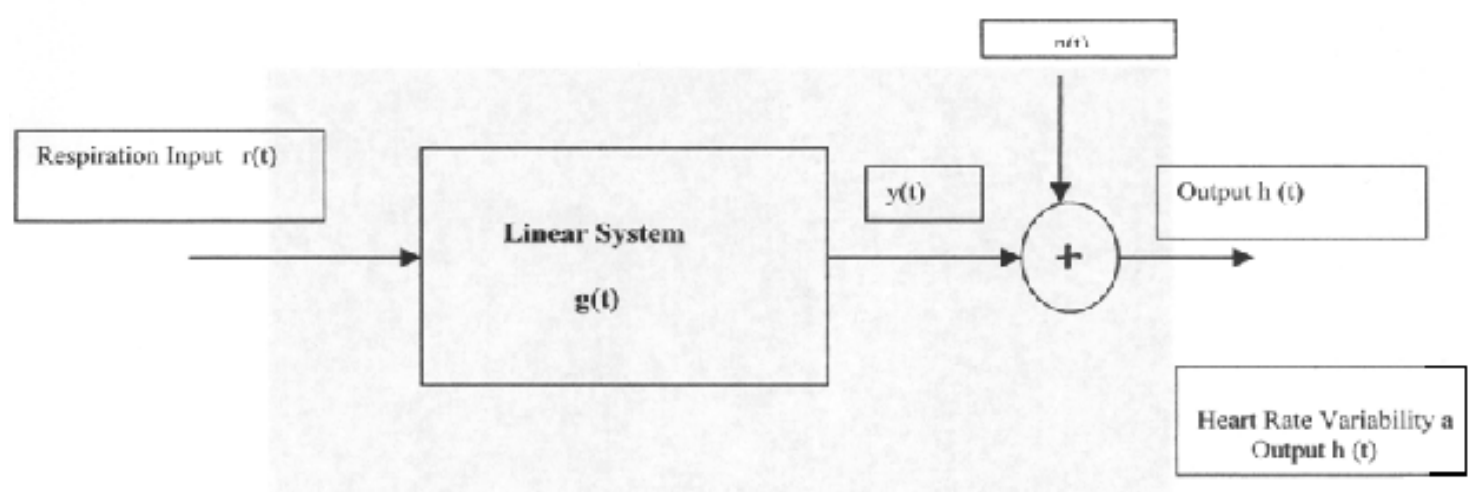

Figure 3: Relationship between input (breathing) and output (HRV signal)

$$
\mathrm{Grh}=\frac{\mathrm{H}}{\mathrm{R}}
$$

And multiply the numerator and denominator of the right side by $\mathrm{R}^{\star}(\mathrm{jw})$,i.e. the complex conjugate of the Fourier transformed input $r(t)$, the result is

$$
\operatorname{Grh}(\mathrm{jw})=\frac{\mathrm{HR}^{*}}{\mathrm{RR}^{*}}=\frac{\operatorname{Prh} *(\mathrm{jw})}{\operatorname{Prr}(\mathrm{w})}
$$

Where $\operatorname{Prh}(\mathrm{jw})$ is the complex conjugate of the cross power spectrum between $\mathrm{r}(\mathrm{t})$ and $\mathrm{h}(\mathrm{t})$ and $\operatorname{Prr}(\mathrm{w})$ is the auto-power spectrum of $r(t)$. Assuming that $n(t)$ and $r(t)$ are not auto-correlated then $\operatorname{Prh}^{\star}(j w)$ is not influenced by the presence of $n(t)$. Since $\operatorname{Prr}(w)$ does not involve $n(t)$ at all, this estimate of $\mathrm{Grh}(\mathrm{jw})$ from $\mathrm{h}(\mathrm{t})$ and $\mathrm{r}(\mathrm{t})$ becomes a good method of overcoming the presence of noise to estimate $\mathrm{H} / \mathrm{R}$ or the frequency gain response.

\section{Results}

The clinical data of fifty two Yoga subjects are summarized in Table 1. There were no significant differences between Yoga practicing and control in these parameters. However, the results of HRV analysis and Approximate Entropy (ApEn) are shown in Figure 4 and Table 2 and Table 3. Figure 4 shows the gain frequency response for both Yoga group and control group at different breathing rates. The gain frequency response of control healthy group is significantly lower $(\mathrm{p}=0.003)$ than Yoga Subjects. Table 2 shows significant difference $(\mathrm{p}=0.001)$ between the value of ApEn of HRV signal at rest for both groups. Table 3 shows the duration of Yoga practicing group and ApEn as well as HRV parameters (heart rate, SDNN (standard deviation of all R-R intervals) at rest.

\begin{tabular}{|c|c|}
\hline Variable & Yoga Subjects $(\mathbf{n = 5 2})$ \\
\hline Age (years), mean \pm SD & $56 \pm 9.6$ \\
\hline BMI $\left(\mathrm{kg} / \mathrm{m}^{2}\right)$ & $22.7 \pm 5.6$ \\
\hline $\begin{array}{c}\text { Mean Yoga practicing duration } \\
\text { (years) }\end{array}$ & 12.4 \\
\hline ESR (mean \pm SD) & $42.6 \pm 25.1$ \\
\hline CRP (mean \pm SD) & $51.4 \pm 124.6$ \\
\hline
\end{tabular}

Table 1: Clinical characteristics of subjects practicing Yoga (BMI, body mass index, CRPC-reactive protein, ESR, erythrocyte sedimentation rate)

\begin{tabular}{|c|c|c|c|}
\hline & Control Group $(\mathbf{n}=\mathbf{5 2})$ & Yoga Group $(\mathbf{n}=\mathbf{5 1})$ & P value \\
\hline ApEn & $0.76 \pm 0.15$ & $0.98 \pm 0.1$ & $<0.05$ \\
\hline Heart rate (beats/sec) & 75.6 & 60.4 & 0.15 \\
\hline SDNN (ms) & $98.6 \pm 36.8$ & $152.3 \pm 18.4$ & 0.003 \\
\hline
\end{tabular}

Table 2: ApEn and HRV parameters (heart rate and SDNN(standard deviation of all R-R intervals) at rest in Yoga and control groups 


\begin{tabular}{|c|c|c|c|c|}
\hline $\begin{array}{c}\text { Duration of Yoga } \\
\text { practicing }\end{array}$ & $\mathbf{1 - 3}$ years $(\mathbf{n}=\mathbf{1 7})$ & $\mathbf{3 - 6}$ years $(\mathbf{n}=\mathbf{2 0})$ & $\mathbf{3 - 6}$ years $(\mathbf{n}=\mathbf{2 0})$ & p value \\
\hline ApEn & $0.88 \pm 0.09$ & $0.92 \pm 0.1$ & $0.95 \pm 0.14$ & 0.001 \\
\hline Heart rate (beats/sec) & $72 \pm 2.6$ & $66 \pm 3.2$ & $59 \pm 2.1$ & NS \\
\hline SDNN (ms) & $95 \pm 8.6$ & $100 \pm 10.3$ & $103 \pm 6.8$ & NS \\
\hline
\end{tabular}

Table 3: HRV parameters (heart rate and SDNN (standard deviation of all R-R intervals) and ApEn at rest in Yoga according to duration of Yoga practicing in years

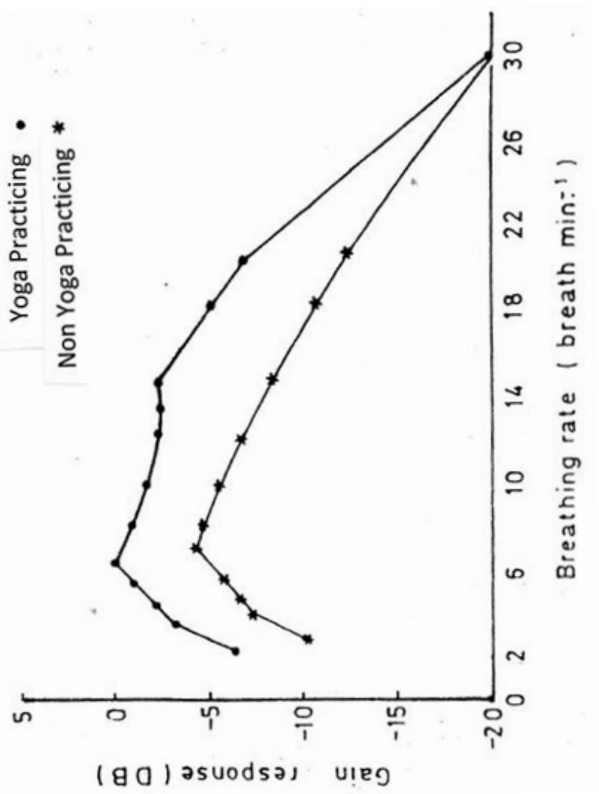

Figure 4: The average frequency gain response of healthy subjects and Yoga subjects at different breathing rates

\section{Discussion}

In this study, we introduced new index ApEn to quantify the regularity of HRV. Lower ApEn indicates less great regularity and less variation of HRV signals and higher ApEn indicates greater regularity and more variation of HRV. Appendix (A) explains the approximate Entropy method. Referring to Table 2, There is significant reduction $(p<0.5)$ in both ApEn and SDNN in Schizophrenia patients group. Also, Table 3 shows significant increase in ApEn index of Yoga subjects related to the duration of the disease. However, SDNN and heart rate did not show significant decrease with the duration of Yoga practicing. This is important finding to quantify the autonomic function of Yoga subjects with respect to their duration of Yoga practicing using ApEn index. In fact, a low ApEn correlates with greater system isolation i.e. less interaction between multiple inputs that makeup a normal control system $[10,13]$. Several normal physiologic processes have been studied to demonstrate the relationship between ApEn and the integrity of the biological system. Kaplan et al. [14] compared healthy young and elderly subjects and found reduced complexity in the elderly. Similarly, Rayan et al. [15] demonstrated ApEn decreased with aging. In this study, we observed the lowest normalized ApEn values in Schizophrenia patients with autonomic dysfunction. Also, we found correlation between increasing ApEn values and Yoga practicing duration as shown in Table 3. The significance of ApEn index is to represent one value (between 0 and 1) which indicates the quality of connection, interaction, regularity and complexity in the system (Appendix A). Applying this concept to the autonomic nervous system of Yoga subjects, it seems their autonomic system has the integrity and interaction with respect to normal healthy subjects which may be manifested in low ApEn values in Healthy normal subjects group as shown in Table 3. The frequency gain response as shown in Figure 4 demonstrates the influence of breathing on gain response with respect to Yoga subjects and healthy subjects. The Yoga subjects group exhibits two distinct peaks at nearly 6 cycles $/ \mathrm{min}(0.1 \mathrm{~Hz})$ and nearly 15 cycles/min $(2.5 \mathrm{~Hz})$. However, healthy subjects group who did not practicing Yoga shows only one lower distinct peak at the same breathing rate 6 cycles/min. It is clear that Yoga subjects show higher gain response to breathing than the healthy subjects at all breathing frequencies. This may be attributed to increase as well as balance in autonomic function especially parasympathetic activities. These observations might be important with respect to the decreased rate of sudden death occurred in Yoga subjects which correlated with increased Vagus (parasympathetic) activity [7-9]. Our finding which correlates the duration of Practicing Yoga group and ApEn may be considered a simple prognostic value to assess the autonomic function of Yoga subjects to screening the autonomic function quantitative way.

\section{Conclusion and Future Work}

This study demonstrates the significance of using the spectral analysis Method and Max Entropy Method in assessing the function of autonomic nervous system for Yoga practicing subjects compared to normal subjects However, further study is required to 
investigate more Yoga elderly subjects as well as using other quantitative methods to identify the prognosis of autonomic function with duration of Yoga.

\section{Acknowledgement}

The author would like to appreciate the cooperation and collaboration of Yoga center, Nashville, TN, USA for facilitating the measurement of physiological signals. I acknowledge the help of Mr. Karim Kamal for his help in statistical analysis of the results of this paper and Mrs. Fatma Ibrahim for Yoga guidance instruction.

\section{References}

1. Fujibayashi M, Matsumoto T, Kishida I, Kimura T, Ishii C, et al. (2009) Autonomic nervous system activity and psychiatric severity in schizophrenia. Psychiatry Clin Neurosci 63: 538-45.

2. Bär KJ, Boettger MK, Koschke M, Schulz S, Chokka P, et al. (2007) Non-linear complexity measures of heart rate variability in acute schizophrenia. Clin Neurophysiol 118: 2009-15.

3. Osipov M, Behzadi Y, Kane JM, Petrides G, Clifford GD (2015) Objective identification and analysis of physiological and behavioral signs of schizophrenia. J Ment Health 24: 276-82.

4. Rajani SN, Indla YR, Archana R, Rajesh P (2015) Role of Yoga on Cardic Autonomic Function Tests and Cognition in Type 2 Diabetes. Int J Res Ayurveda Pharm 6: 764-6.

5. Chu IH, Lin YJ, Wu WL, Chang YK, Lin IM (2015) Effects of Yoga on Heart Rate Variability and Mood in Women: A Randomized Controlled Trial. J Altern Complement Med 21: 789-95.

6. Posadzki P, Kuzdzal A, Lee MS, Ernst E (2015) Yoga for Heart Rate Variability: A Systematic Review and Meta-analysis of Randomized Clinical Trials. Appl Psychophysiol Biofeedback 40: 239-49.

7. Chang JS, Hwang SS, Yi SH, Kim Y, Lee YS, et al. (2015) Evaluating subjective domains of antipsychotic-induced adverse effects using heart rate variability. Psychiatry Clin Neurosci 69: 283-91.

8. Koschke M, Boettger MK, Schulz S, Berger S, Terhaar J, et al. (2009) Autonomy of autonomic dysfunction in major depression. Psychosom Med 71: 852-60.

9. Birkhofer A, Schmidt G, Förstl H (2005) [Heart and brain -- the influence of psychiatric disorders and their therapy on the heart rate variability]. Fortschr Neurol Psychiatr 73: 192-205.

10. Zahn TP (1988) Studies of autonomic psychophysiology and attention in schizophrenia. Schizophr Bull 14: 205-8.

11. Pincus SM (1991) Approximate entropy as a measure of system complexity. Proc Natl Acad Sci USA 88: $2297-301$.

12. Pincus SM (1994) Greater signal regularity may indicate increased system isolation. Math Biosci 122: 161-81.

13. Pincus SM, Goldberg AL (1994) Physiological time series analysis: What does regularity quantify? Am J Physiol 266: H1643-56.

14. Kaplan DT, Furman MI, Pincus SM, Rayan SM, Lipsitz LA, et al. (1991) Aging and the complexity of cardiovascular dynamics. Biophys J 59: 945-9.

15. Rayan SM, Goldberg AL, Pincus SM, Mietus J, Lipsitz LA (1994) Gender and age related differences in heart rate dynamics: are women more complex than men? J Am Coll Cardiol 24: 1700-7.

16. Pincus SM, Gladstone IM, Ehrenkranz MS (1991) A regularity statistics for medical data analysis. J Clin Monit 7: $335-45$.

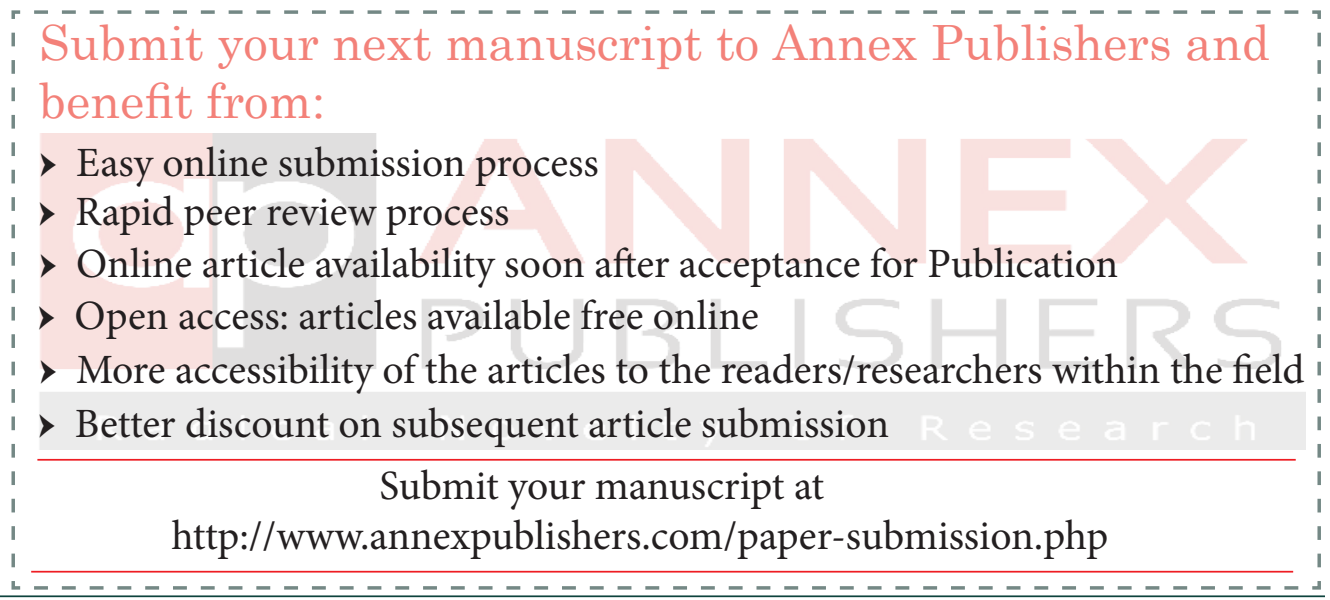

